\title{
Multifocal electroretinogram in children on atropine treatment for myopia
}

\author{
C D Luu, A M I Lau, A H C Koh, D Tan
}

Br J Ophthalmol 2005;89:151-153. doi: 10.1136/bjo.2004.045526

Aim: To assess retinal function by multifocal electroretinogram (mfERG) in children on atropine eye drops for the treatment of myopia.

Methods: mfERGs were recorded in children receiving atropine eye drops $(n=48)$ once daily for 2 years and in those receiving placebo eye drops $(n=57)$ for a similar time. All recordings were performed between the second and third month of cessation of atropine/placebo treatment by a masked investigator. The amplitude and implicit time of the first order kernel (k1) and first slice of the second order kernel (k21) of mfERG responses were used to study the outer and inner retinal function, respectively.

Results: There was no significant reduction in $\mathrm{kl}$ response amplitudes of the atropine group compared to that of the placebo group (N1, $\mathrm{p}=0.181 ; \mathrm{Pl}, \mathrm{p}=0.150$ ). No significant difference in the $k 1$ response implicit times between the groups was found (N1, $p=0.767 ; \mathrm{P} 1, p=0.849$ ). The differences in the $k 21$ amplitudes and implicit times between the groups were not statistically significant (k21 amplitude, $p=0.058 ; k 21$ implicit time, $p=0.156$ ).

Conclusions: Daily atropine usage over 2 years for the treatment of myopia has no significant effect on retinal function as demonstrated by recordings of mfERG.

M uscarinic transmission in retinal cells is believed to be involved in the visual control of refractive development. Experimental studies have revealed that the non-selective muscarinic antagonist, atropine, reduces the progression of axial myopia in humans via a nonaccommodative mechanism. ${ }^{2}$ A recent study showed that topically applied atropine solution ( $1 \%$ ) on a daily basis reduced the mean annual rate of myopia progression from approximately $0.77 \mathrm{D}$ per year to $0.05 \mathrm{D}$ per year during the treatment period. ${ }^{3}$ However, the actual mechanisms involved in the process are still unclear.

Although the beneficial effect of atropine in reducing progression of myopia in humans has been reported in a number of studies, ${ }^{3-5}$ the effects of atropine treatment on retinal function have not been studied previously. There are at least two potential chronic side effects associated with the long term use of atropine. Firstly, accumulation of atropine over a period of time might be toxic to the neural retina. Secondly, constant pupillary dilatation will increase the amount of light entering the eye and could theoretically cause photic damage to the retina. Photic retinopathy has been reported from a variety of light sources and intensities including solar exposure, welding arc, laser light, operating microscope, ophthalmoscope and fundus camera exposure. ${ }^{67}$ Infants and adolescents are at a greater risk of photic retinopathy because of their clear ocular media. ${ }^{7}$
The multifocal electroretinogram (mfERG), first described by Sutter and Tran, ${ }^{8}$ has proved to be a sensitive technique in the detection of retinal dysfunction. ${ }^{9}$ In this study, we used the mfERG to assess the effect of atropine usage on retinal function.

\section{METHODS \\ Subjects}

The ATOM (Atropine in the Treatment of Myopia) study that is currently being conducted at the Singapore Eye Research Institute aims to quantify the efficacy of atropine in controlling myopia progression in children as well as to assess the safety of atropine treatment. Subjects enrolled in the ATOM study were randomly allocated into two groups where they received either atropine (atropine sulphate $1 \%$ ) or placebo (Isoptotears) eye drops. The eye to receive the eye drops was also randomly selected for each child. Only the randomised eye received the eye drops once daily for 2 years, while the contralateral eye served as an untreated control. Subjects who had completed the treatment with good compliance were recruited for the present study. Parental informed consent was obtained for all subjects before their participation. The current study was approved by the human ethics committee of Singapore Eye Research Institute.

\section{mfERG recording}

The recordings were performed between the second and third month (mean (SD) 2.6 (0.43) months) of cessation of atropine/placebo treatment. All mfERG recordings were performed by one investigator who was masked to the treatment status. The mfERG was recorded using DTL fibre electrodes (Diagnosys LLC, Littleton, MA, USA) and followed the guidelines recommended by the International Society for Clinical Electrophysiology of Vision (ISCEV) standards. $^{10}$ Pupils were dilated using tropicamide $1 \%$ and mfERG recording was commenced when both pupils were equally dilated to at least $7 \mathrm{~mm}$. The testing stimulus comprised 37 retinally scaled hexagons, which were randomly alternated between white and black frames on a monochrome monitor at a rate of $75 \mathrm{~Hz}$. The intensities for the white and black frames were 2.66 cd.s.m ${ }^{-2}$ and 0.02 cd.s.m ${ }^{-2}$, respectively. Background luminance was $100 \mathrm{~cd} . \mathrm{m}^{-2}$. Recorded signals were band pass filtered between $3-100 \mathrm{~Hz}$ and amplified 100000 times. Only the central 28 degrees retina was tested and both eyes were recorded concurrently. The duration of the recording was approximately 4 minutes, which was divided evenly into slightly overlapping 16 segments of about 14 seconds each for patients' comfort.

Abbreviations: ATOM study, Atropine in the Treatment of Myopia study; ISCEV, International Society for Clinical Electrophysiology of Vision; mfERG, multifocal electroretinogram 

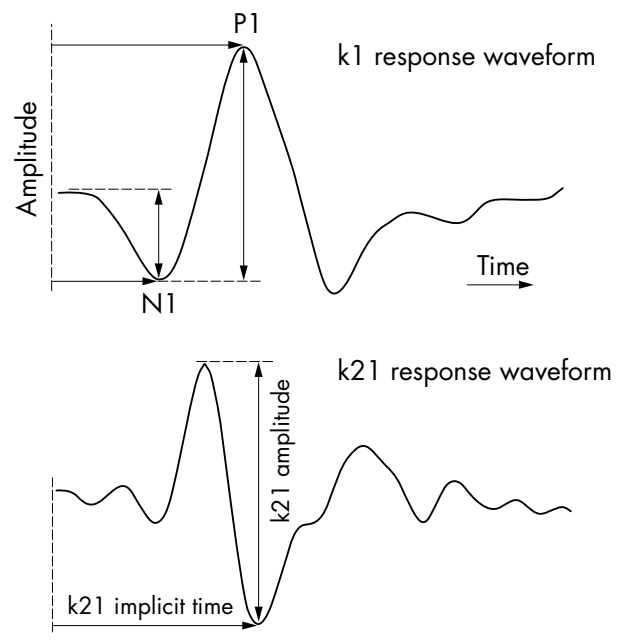

Figure 1 The waveforms of the first order $(\mathrm{k} 1)$ and the first slice of the second order kernel (k21) mfERG response. The vertical arrows represent the amplitude measurement. The horizontal arrows represent the response implicit time measurement.

\section{Analysis}

The waveforms of the summed responses from 37 hexagonal elements of the first order (kl) and first slice of second order (k21) kernel of the mfERG were generated using the VERIS software (VERIS Science 4.x, Electro-Diagnostic Imaging, San Mateo, CA, USA). Previous studies on primates have suggested that the first negative trough $(\mathrm{Nl})$ of the $\mathrm{kl}$ response is shaped by the activities of both photoreceptors and OFF bipolar cells. ${ }^{11}$ The contributions to the positive peak (Pl) of the kl response are predominantly from the ON bipolar cells. The $\mathrm{k} 2 \mathrm{l}$ response derives from adaptive mechanisms and thus reflects the inner retinal function. ${ }^{11}$ The response amplitude and implicit time of $\mathrm{Nl}$ and $\mathrm{Pl}$ as well as the k2l response were measured and used for the analysis (fig 1). For comparison, the mfERG data were subdivided into four categories; atropine treated eye, atropine untreated fellow eye, placebo treated eye and placebo untreated fellow eye. An analysis of variance (ANOVA) test was used to indicate the significance level of response amplitude and implicit time differences between categories.

\section{RESULTS}

There was no significant difference in the demographic data between the atropine and placebo groups at the time of mfERG testing. The mean age of atropine and placebo groups were 11.6 and 11.4 years, respectively. There was no significant difference in the visual acuity among the four categories of eyes (table 1). The atropine treated eye was significantly less myopic than the placebo treated eye $(p<0.001)$. A summary of demographic data, duration of treatment, visual acuity, and refraction of the study subjects is shown in table 1 .

A summary of the mfERG parameters for all four categories is shown in table 2. The mean $\mathrm{Nl}$ and $\mathrm{Pl}$ amplitudes of the atropine treated and untreated fellow eyes were slightly reduced compared to those in the placebo treated and untreated fellow eyes. The differences in $\mathrm{Nl}$ and $\mathrm{Pl}$ amplitudes among the four categories of eyes, however, were not statistically significant (table 2 ). There were no significant differences in the mean implicit times of $\mathrm{Nl}$ and $\mathrm{Pl}$ responses of the eyes in the atropine and placebo groups (table 2).

The mean k21 response amplitude in the atropine group was also slightly reduced compared to that in the placebo group but the apparent reduction did not reach significance $(p=0.058)$. The difference in $\mathrm{k} 2 \mathrm{l}$ amplitude between the atropine treated and untreated fellow eye was not statistically significant $\left(\mathrm{t}_{(94)}=0.35 \mathrm{l}, \mathrm{p}=0.726\right)$. There were no significant differences in the mean k21 implicit times among the four categories of eyes.

\section{DISCUSSION}

High myopia is associated with sight threatening retinal complications and interventions to slow myopia progression in children would seem to be beneficial. To date, only atropine eye drops have been shown to have a consistent effect on the retardation of myopia progression. ${ }^{12}{ }^{13}$ Safety data on the effect of atropine on visual function have been reported in previous clinical trial studies but only in the form of best corrected visual acuity. We are unaware of previous reports on the possible effect of atropine on retinal function using electrophysiological technique. The electrophysiological findings from the current study showed that atropine use over 2 years has no significant effect on retinal function. Since the retinal function is not significantly altered soon after cessation of atropine treatment when the effect or cumulative effect of atropine on retinal function, if there is any, is expected to be highest, it is very unlikely that atropine induced retinal dysfunction will occur in later years because the concentration of atropine in the retina will decrease over time.

The difference in k2l amplitude between groups was almost reaching statistical significance $(p=0.058)$, suggesting that there may be a trend towards reduced $\mathrm{k} 2 \mathrm{l}$ amplitude in atropine treated eyes. However, the mean k2 1 amplitude of the atropine treated eye was greater than that of the atropine fellow eye. If the trend exists, we would expect that the k2 1 response of the atropine treated eye to have smaller amplitudes than that of the fellow eye.

In conclusion, the findings from this study show that atropine usage over 2 years for the treatment of myopia causes no significant retinal dysfunction as demonstrated by the use of the multifocal ERG technique.

\begin{tabular}{|c|c|c|c|}
\hline & $\begin{array}{l}\text { Atropine group } \\
(\mathrm{n}=48)\end{array}$ & $\begin{array}{l}\text { Placebo group } \\
(n=57)\end{array}$ & $\mathrm{p}$ Value \\
\hline Age (years) (mean (SD)) & $11.6(1.4)$ & $11.4(1.4)$ & $0.734^{*}$ \\
\hline Sex (females/males) & $27 / 21$ & $27 / 30$ & $0.364^{* *}$ \\
\hline Duration of treatment (years) (mean (SD)) & $2.001(0.03)$ & $1.993(0.03)$ & $0.179^{*}$ \\
\hline LogMAR VA of treated eye (mean (SD)) & $0.002(0.04)$ & $0.018(0.05)$ & $0.155^{*}$ \\
\hline LogMAR VA of fellow eye (mean (SD)) & $0.007(0.04)$ & $0.012(0.04)$ & $0.327^{*}$ \\
\hline Refraction of treated eye (mean (SD)) & $-2.50(1.39) \mathrm{D}$ & $-4.65(1.22) \mathrm{D}$ & $<0.001^{*}$ \\
\hline Refraction of fellow eye (mean (SD)) & $-4.04(1.52) \mathrm{D}$ & -4.61 (1.32) D & $0.022^{*}$ \\
\hline
\end{tabular}


Table 2 The mfERG parameters of the atropine and placebo groups

\begin{tabular}{|c|c|c|c|c|c|}
\hline \multirow{2}{*}{$\begin{array}{l}\text { mfERG } \\
\text { parameters }\end{array}$} & \multicolumn{2}{|c|}{ Atropine group $(n=48)$} & \multicolumn{2}{|c|}{ Placebo group $(n=57)$} & \multirow[b]{2}{*}{ p Value } \\
\hline & Treated eye & Fellow eye & Treated eye & Fellow eye & \\
\hline $\begin{array}{l}\text { N1 amplitude } \\
\text { Mean (SD) }\end{array}$ & $599(124)$ & $595(125)$ & $644(1.58)$ & & \\
\hline Pl amplitude & (.) & (1) & $0.44(1.00)$ & 0.4011 .721 & 0.101 \\
\hline Mean (SD) & $16.61(3.04)$ & $16.58(3.26)$ & $17.85(4.37)$ & $17.87(4.59)$ & 0.150 \\
\hline Mean (SD) & $4.09(0.97)$ & $4.02(0.99)$ & $4.47(1.23)$ & $4.53(1.39)$ & 0.058 \\
\hline N1 implicit time & & & & & \\
\hline $\begin{array}{c}\text { Mean (SD) } \\
\text { P1 implicit time }\end{array}$ & $15.65(0.73)$ & $15.56(0.67)$ & $15.56(0.66)$ & $15.65(0.66)$ & 0.767 \\
\hline Mean (SD) & $29.00(0.93)$ & $28.93(0.97)$ & $29.14(0.99)$ & $29.03(0.84)$ & 0.849 \\
\hline K21 implicit time & & & & & \\
\hline Mean (SD) & $30.50(1.99)$ & $30.62(1.32)$ & $30.83(1.53)$ & $31.15(1.32)$ & 0.156 \\
\hline
\end{tabular}

\section{ACKNOWLEDGEMENTS}

The authors thank Professor Wallace Foulds for his constructive comments. The study was supported by a pilot grant from the Singapore National Medical Research Council (R285/29/2002).

\section{Authors' affiliations}

C D Luu, A M I Lau, D Tan, Singapore Eye Research Institute, Singapore D Tan, Singapore National Eye Centre, Singapore

Grant support: A pilot grant from the Singapore National Medical Research Council.

Correspondence to: Dr Chi D Luu, Singapore Eye Research Institute, 11 Third Hospital Avenue, Singapore 168751; seri_chiluu@snec.com.sg

Accepted for publication 13 June 2004

\section{REFERENCES}

1 Stone RA, Lin T, Laties AM. Muscarinic antagonist effects on experimental chick myopia. Exp Eye Res 1991;52:755-8.

2 McBrien NA, Moghaddam HO, Reeder AP. Atropine reduces experimental myopia and eye enlargement via a nonaccommodative mechanism. Invest Ophthalmol Vis Sci 1993;34:205-15.
3 Kennedy RH, Dyer JA, Kennedy MA, et al. Reducing the progression of myopia with atropine: a long term cohort study of Olmsted County students. Binocul Vis Strabismus Q 2000;15:281-304.

4 Shih YF, Chen $\mathrm{CH}$, Chou $\mathrm{AC}$, et al. Effects of different concentrations of atropine on controlling myopia in myopic children. J Ocul Pharmacol Ther 1999; 15:85-90.

5 Yen MY, Liu JH, Kao SC, et al. Comparison of the effect of atropine and cyclopentolate on myopia. Ann Ophthalmol 1989;21:180-2, 187.

6 Lessel M, Thaler A, Heilig P, et al. Intraoperative retinal light damage reflected in electrophysiologic data. Doc Ophthalmol 1991;76:323-33.

7 Lawwill T, Crockett S, Currier G. Retinal damage secondary to chronic light exposure, thresholds and mechanisms. Doc Ophthalmol 1977;44:379-402.

8 Sutter EE, Tran D. The field topography of ERG components in man-I. The photopic luminance response. Vis Res 1992;32:433-46.

9 Hood DC. Assessing retinal function with the multifocal technique. Prog Retin Eye Res 2000; 19:607-46.

10 Marmor MF, Hood DC, Keating D, et al. Guidelines for basic multifocal electroretinography (mfERG). Doc Ophthalmol 2003;106:105-15.

11 Hood DC, Frishman U, Saszik S, et al. Retinal origins of the primate multifocal ERG: implications for the human response. Invest Ophthalmol Vis Sci 2002;43:1673-85.

12 Saw SM, Gazzard G, Au Eong KG, et al. Myopia: attempts to arrest progression. Br J Ophthalmol 2002;86:1306-11.

13 Saw SM, Shih-Yen EC, Koh A, et al. Interventions to retard myopia progression in children: an evidence-based update. Ophthalmology 2002;109:415-21 